\title{
A FUNDAMENTAL PROBLEM IN LINEAR INEQUALITIES WITH APPLICATIONS TO THE TRAVELLING SALESMAN PROBLEM *
}

\author{
Katta G. MURTY \\ University of Michigan, Ann Arbor, Michigan, U.S.A. \\ Received 5 November 1970 \\ Revised manuscript received 29 November 1971
}

We consider a system of $m$ linearly independent equality constraints in $n$ nonnegative variables: $A x=b, x \geqq 0$. The fundamental problem that we discuss is the following: suppose we are given a set of $r$ linearly independent column vectors of $A$, known as the special column vectors. The problem is to develop an efficient algorithm to determine whether there exists a feasible basis which contains all the special column vectors as basic column vectors and to find such a basis if one exists. Such an algorithm has several applications in the area of mathematical programming. As an illustration, we show that the famous travelling salesman problem can be solved efficiently using this algorithm. Recent published work indicates that this algorithm has applications in integer linear programming. An algorithm for this problem using a set covering approach is described.

\section{Introduction}

1.1. We consider the system of linear equality constraints in nonnegative variables

$$
\begin{gathered}
A x=b, \\
x \geqq 0,
\end{gathered}
$$

where $A$ is a matrix of order $m \times n, b$ a column vector of order $m \times 1$, and $x$, the column vector of variables, is of order $n \times 1$. Without any loss of generality we assume that $A$ has rank $m$ and that $n>m$.

* This research has been partially supported by the ISDOS research project and the National Science Foundation under Grant GK-27872 with the University of Michigan. 
If $D$ is any matrix we will denote by $D_{j}$ the $j$-th column vector of $D$.

A basis for (1) is any square nonsingular submatrix $B$ of $A$, of order $m \times m$. A basis $B$ is said to be a feasible basis for (1), (2) if $B^{-1} b \geqq 0$. A basis which is not feasible is said to be an infeasible basis for (1). While considering a basis

$$
B=\left(A_{{ }_{j_{1}}} \vdots \cdots \vdots A_{{ }_{j_{m}}}\right)
$$

for (1), the column vectors in $B$ will be referred to as basic column vectors and the variables $\left(x_{j_{1}}, \ldots, x_{j_{m}}\right)$ associated with them as basic variables. Let $x_{B}=\left(x_{j_{1}}, \ldots, x_{j_{m}}\right)^{\mathrm{T}}$. All the remaining variables in (1) and their associated column vectors will be termed nonbasic when considering the basis $B$. The basic solution of (1) corresponding to the basis $B$ is obtained by setting

$$
\begin{aligned}
& \text { all nonbasic variables }=0, \\
& \text { basic vector } x_{B}=B^{-1} b .
\end{aligned}
$$

Thus $B$ is a feasible basis for (1), (2) if the basic solution of (1) corresponding to the basis $B$ also satisfies the nonnegativity constraints (2).

1.2. Suppose we are given a subset of $r$ linearly independent column vectors of $A$, say $A_{{ }_{1}}, \ldots, A_{r}$, which are known as the special column vectors. The variables, $x_{1}, \ldots, x_{r}$, in (1) associated with them are known as the special variables.

The problem that we will consider is that of developing an efficient algorithm to settle whether there exists a feasible basis for (1) which contains all the special column vectors and to find such a basis if one exists.

1.3. The total number of bases for (1) which contain all the special column vectors is $\leqq(n-r) ! /[(m-r) !(n-m) !]$. So the problem posed in 1.2 can be solved in a finite number of steps by checking for feasibility every one of the bases which contain all the special column vectors. However this method is not practicable unless $n-m$ and $m-r$ are very small, as otherwise, the computational effort for doing this becomes 
prohibitive. As a justification for calling this a fundamental problem, we now show that given a travelling salesman problem and a real number $\beta$, the problem of finding a tour of the salesman whose total cost is $\leqq \beta$, if such tours exist, or proving that the total cost corresponding to every possible tour is $>\beta$, can be formulated as a problem of the type posed in 1.2. We also discuss some other applications for an algorithm for solving a problem of the type posed in 1.2.

\section{Application to the travelling salesman and other problems}

2.1. The well-known travelling salesman problem may be stated as follows. We are given a set of $N$ cities, $N \geqq 2 . C_{i j}$, the cost of travelling from city $i$ to city $j$, is given for all $i \neq j$. A salesman wishes to start from some city, visit each of the remaining cities in the set once and only once and at the end return to the city he started from. The problem is to determine the optimal order for visiting the cities.

2.2. Suppose he starts at city $i_{1}$. From $i_{1}$ suppose he goes to city $i_{2}$ and in general from city $i_{r}$ he goes to $i_{r+1}, r=1$ to $N-1$, and then in the end he returns to $i_{1}$. Then the order in which the cities are visited is

$$
i_{1} i_{2} \ldots i_{N} i_{1},
$$

where $\left(i_{1}, \ldots, i_{N}\right)$ is a permutation of $1,2, \ldots, N$. Such an order of visiting the cities is known as a tour covering the cities $1,2, \ldots, N$. We will use the brief term "tour" to indicate "a tour covering the cities $1,2, \ldots$, $N$ " and we will use the letter " $t$ " to denote tours. If $F$ is a proper subset of the set of cities $1,2, \ldots, N$ whose cardinality is at least two, then any tour covering only the cities in the subset $F$ will be known as a "subtour covering the subset of cities F" or just as a "subtour". The total cost of the tour in (3) is

$$
\sum_{r=1}^{N-1} C_{i_{r} i_{r+1}}+C_{i_{N} i_{1}} .
$$


The ordered pairs $\left(i_{1}, i_{2}\right), \ldots,\left(i_{N}, i_{1}\right)$ are called the arcs or cells in the tour in (3). Since the arcs completely specify the order in which the cities are visited, the tour $t$ in (3) may be represented by the set of arcs in it, as in

$$
t=\left\{\left(i_{1}, i_{2}\right), \ldots,\left(i_{N}, i_{1}\right)\right\}
$$

Because of the cyclic nature of the tour, the starting city is immaterial. Thus there are $(N-1)$ ! tours and the problem is to develop an algorithm for finding the minimal cost tour efficiently.

2.3. Another way of representing a tour is to define a matrix $X=\left(x_{i j}\right)$ corresponding to it, where

$$
\begin{aligned}
x_{i j} & =1 \quad \text { if the salesman travels from } i \text { to } j \text { in the tour }, \\
& =0 \quad \text { otherwise } .
\end{aligned}
$$

The matrix $X$ corresponding to any tour $t$ as defined by (5) is a special kind of permutation matrix known as a cyclic permutation matrix. The cost corresponding to the tour represented by the cyclic permutation matrix $X$ is

$$
z(X)=\sum_{i=1}^{N} \sum_{\substack{j=1 \\ j \neq i}}^{N} C_{i j} x_{i j}
$$

2.4. In general any square matrix of order $N \times N$ which consists of a single nonzero entry equal to 1 in each of its rows' and columns is known as a permutation matrix or an assignment of order $N$. Since we only deal with assignments of order $N$ here, we will refer to them briefly as just assignments.

All the assignments are the extreme points of the set of all feasible solutions to the system of constraints 


$$
\begin{gathered}
\sum_{i=1}^{N} x_{i j}=1, j=1 \text { to } N, \\
\sum_{j=1}^{N} x_{i j}=1, i=1 \text { to } N, \\
x_{i j} \geqq 0 \text { for all } i, j
\end{gathered}
$$

and conversely every basic feasible solution of (7), (8) is an assignment [5]. The set of all feasible solutions to (7), (8) is denoted by $K_{A}$ and it is the convex hull of all the permutation matrices of order $N \times N$. In particular the permutation matrix represented by the $N \times N$ identity matrix $I$ is known as the diagonal assignment or D.A. A permutation matrix is completely specified if we specify the cells $(i, j)$ in it which have unit entries in them, since all the remaining cells have zero entries. Thus if the permutation matrix $X$ has unit entries in the cells $\left(r, j_{r}\right)$, $r=1$ to $N$, we will find it convenient to represent $X$ by

$$
X=\left\{\left(1, j_{1}\right), \ldots,\left(N, J_{N}\right)\right\} \text {, }
$$

where $\left(j_{1}, \ldots, j_{N}\right)$ is a permutation of $1,2, \ldots, N$.

We will denote assignments by $a_{1}, a_{2}$ etc.

2.5. Since the travelling salesman problem requires that the salesman should visit each city once and only once, the cells of the form $(i, i)$ in the cyclic permutation matrix corresponding to any tour would always contain a zero entry.

Thus when dealing with the travelling salesman problem we can define the diagonal entries $C_{i i}$ in the cost matrix arbitrarily and this will not affect our problem of the validity of equation (6) for tours. We let $C_{i i}=\delta$ for $i=1$ to $N$ where $\delta$ is a sufficiently large positive number to be determined later on. Then $C=\left(C_{i j}\right)$ is known as the cost matrix for the travelling salesman problem.

2.6. Without any loss of generality we assume that all $C_{i j} \geqq 0$, since the set of optimal tours to the travelling salesman problem remains unchanged if we add an arbitrary constant to each element in a row or column of the cost matrix $C$. So $C \geqq 0$. 
2.7. If a permutation matrix $X$ has a unit entry in the cell $(i, i):$ i.e. if $x_{i i}=1$, then it is said to have a self-loop at city $i$. Any cell of the form $(i, i)$ is called a diagonal cell and all the other cells $(i, j), i \neq j$, are called nondiagonal cells.

A non-tour is an assignment which is not a tour and which has no self-loops. In other words it is an assignment without any unit entries among the diagonal cells, whose unit cells constitute at least two subtours.

We will now show that the travelling salesman problem can be solved by solving a problem of the type discussed in section 1.2. For this we will use the results in [9].

2.8. Lemma. Suppose $\left\{\left(i_{1}, j_{1}\right), \ldots,\left(i_{N}, j_{N}\right)\right\}$ is an assignment and the variables

$$
\left\{x_{11}, \ldots, x_{N N} ; x_{i_{1} j_{1}}, x_{i_{2} j_{2}}, \ldots, x_{i_{N} j_{N}}\right\}
$$

are such that the rank of the set of column vectors corresponding to them in $(7)$ is $(2 N-1)$. Then $\left\{\left(i_{1}, j_{1}\right), \ldots,\left(i_{N}, j_{N}\right)\right\}$ must be a tour and vice versa.

The proof of this lemma is very simple. It is contained in the proof of Theorem 1 in [9].

2.9. Theorem. The set of all adjacent extreme points of the D.A. on the convex polytope $K_{A}$, the set of all feasible solutions to (7), (8), consists of

(i) all the tours and

(ii) all subtours covering a proper subset of the cities in $1, \ldots, N$ with self-loops at the remaining cities.

All the non-tours are not adjacent extreme points of the D.A. on $K_{A}$.

This is Theorem 1 in [9] and it follows easily from 2.8. This theorem is also a corollary of a theorem of Heller [8] .

2.10. Suppose we pick $C_{i i}=\delta$ where $\delta$ is some positive number very much larger than the sum of the $N$ largest numbers among the $C_{i j}, i \neq j$. 
Let $\beta$ be any real number less than the cost of some tour. Consider the system of constraints

$$
\begin{aligned}
& \sum_{i=1}^{N} x_{i j}=1, \quad j=1 \text { to } N, \\
& \sum_{j=1}^{N} x_{i j}=1, \quad i=1 \text { to } N, \\
& z(X)=\sum_{i=1}^{N} \sum_{j=1}^{N} C_{i j} x_{i j}=\beta, \\
& x_{i j} \geq 0 \text { for all } i, j,
\end{aligned}
$$

obtained by adding the new constraint

$$
z(X)=\beta,
$$

to (7) and (8).

\subsection{Theorem. If}

$$
\left\{x_{11}, x_{22}, \ldots, x_{N N}, x_{i_{1} j_{1}}, \ldots, x_{i_{N} j_{N}}\right\}
$$

is a feasible basis for $(9),(10)$, then $\left\{\left(i_{1}, j_{1}\right), \ldots,\left(i_{N}, j_{N}\right)\right\}$ must be a tour whose cost is $\leqq \beta$.

Proof. The total number of linearly independent equality constraints in (9) is $2 N$. Hence if $\left\{x_{11}, \ldots, x_{N N}, x_{i_{1} j_{1}}, \ldots, x_{i_{N} j_{N}}\right\}$ is a basis for (9), the rank of the set of column vectors corresponding to these variables in (9) must be $2 N$. Hence the rank of the set of column vectors of the same variables in $(7)$ must be $(2 N-1)$. This implies by Lemma 2.8 that if $\left\{\left(i_{1}, i_{1}\right), \ldots,\left(i_{N}, j_{N}\right)\right\}$ is an assignment, then it must be a tour.

By Theorem 4.1 of [10] every basic feasible solution of (9), (10) must either be a basic feasible solution of (7) lying on the hyperplane (11) (in this case $\left\{\left(i_{1}, j_{1}\right), \ldots,\left(i_{N}, j_{N}\right)\right\}$ must be an assignment and hence 
a tour of cost $\beta$ ) or it must be the intersection of some edge of the convex polytope $K_{A}$ with the hyperplane (11). In this case, the set of variables $\left\{x_{11}, \ldots, x_{N N} ; x_{i_{1} j_{1}}, \ldots, x_{i_{N} j_{N}}\right\}$ must contain two assignments, say $a_{1}$ and $a_{2}$ which are adjacent to each other on $K_{A}$ such that the basic feasible solution corresponding to the given basis,

$$
\left\{x_{11}, \ldots, x_{N N}, x_{i_{1} j_{1}}, \ldots, x_{i_{N} j_{N}}\right\}
$$

to (9), (10) is a convex combination of the assignments $a_{1}$ and $a_{2}$. This implies that $\beta$ is a convex combination of $z\left(a_{1}\right)$ and $z\left(a_{2}\right)$. We selected $\beta$ to be less than the cost of some tour and $\delta=C_{i i}$ for any $i$, to be much larger than the sum of the $N$ largest numbers among the $C_{i j} \geq 0$, $i \neq j$.

If both the assignments $a_{1}$ and $a_{2}$ contained at least one diagonal cell, then both $z\left(a_{1}\right) \geqq \delta$ and $z\left(a_{2}\right) \geq \delta$. This implies that $\beta$ cannot be a convex combination of $z\left(a_{1}\right)$ and $z\left(a_{2}\right)$ since $\beta<\delta$. So at least one of the assignments $a_{1}$ or $a_{2}$ must not contain any diagonal cells and its cost must be $\leqq \beta$. This implies that the assignment must be $\left\{\left(i_{1}, j_{1}\right), \ldots\right.$, $\left.\left(i_{N}, j_{N}\right)\right\}$ and since the rank of the column vectors corresponding to $\left\{x_{11}, \ldots, x_{N N}, x_{i_{1} j_{1}}, \ldots, x_{i_{N} j_{N}}\right\}$ in (7) is $2 N-1,\left\{\left(i_{1}, j_{1}\right), \ldots,\left(i_{N}, j_{N}\right)\right\}$ must be a tour. Hence it is a tour with cost $\leqq \beta$.

Conversely if $\left\{\left(i_{1}, j_{1}\right), \ldots,\left(i_{N}, j_{N}\right)\right\}$ is a tour with cost $\leqq \beta$, then by Theorem 2.9 here and 4.1 of [10] we conclude that $\left\{x_{11}, \ldots, x_{N N}\right.$, $\left.x_{i_{1} j_{1}}, \ldots, x_{i_{N} j_{N}}\right\}$ must be a feasible basis for (9) and (10).

2.12. Corollary. Let the column vectors of $x_{11}, \ldots, x_{N N}$ in (9) be the special column vectors. Then from 2.11 we conclude that (9), (10) have a feasible basis which contains all these special column vectors as basic column vectors if and only if there exists a tour whose cost is $\leqq \beta$. Also in any such basis, the remaining variables (excluding the special ones which are the diagonal variables $x_{i i}, i=1$ to $N$ ) provide the tour whose cost is $\leqq \beta$.

Thus we have transformed the problem of finding a tour with cost $\leqq \beta$ into a problem of the type 1.2 . So the algorithm for problems of the type 1.2 can be used to find a tour of $\operatorname{cost} \leqq \beta$, if one exists.

By reducing the value of $\beta$ successively and applying the algorithm, we can generate tours whose cost decreases. We terminate when the minimal cost tour is obtained. 


\subsection{Applications in integer programming.}

Recently Cabot and Hurter [4] Glover [6] and Raghavachari [12] have discussed algorithms for integer programming problems which require the solving of several problems of the type posed in 1.2. So an efficient algorithm for solving problems of the type posed in 1.2 will have applications in integer programming as well.

\section{Algorithm for solving the fundamental problem}

3.1. We again consider the system

$$
\begin{gathered}
A x=b, \\
x \geq 0,
\end{gathered}
$$

where $A$ is a matrix of order $m \times n$ and rank $m$. The variables $x_{1}, \ldots, x_{r}$ are the specified special variables and we know that $\left\{A_{\cdot_{1}}, \ldots, A_{r}\right\}$ is a linearly independent set.

A feasible basis for (1), (2) which contains all the special column vectors as basic column vectors will be known as a desired basis. We wish to either conclude that no desired basis exists or to find one if it does. In this section we let $\Gamma=\{1, \ldots, n\}$. If $\Delta \subset \Gamma$ then $A_{\Delta}$ denotes the submatrix of $A$ whose column vectors are $\left\{A_{\cdot j}: j \in \Delta\right\}$. Also, if $D$ is a submatrix of $A$ of order $m \times k$, consisting of $k$ column vectors of $A$, then $\nu(D)=\left\{j: A_{\cdot j}\right.$ is in the submatrix $\left.D\right\}$. So $\nu(D)$ is a subset of $\Gamma$ of cardinality $k$. We will define a class $\theta$ of nonempty subsets of $\Gamma$. As we progress in the algorithm we will augment this class $\theta$ with additional nonempty subsets of $\Gamma$ at each stage. We use symbols like " $c_{p}$ " to denote the subsets in the class $\theta$.

Initially let

$$
c_{p}=\{p\} \quad \text { for } p=1 \text { to } r
$$

and let $\theta=\left\{c_{1}, \ldots, c_{r}\right\}$ at this stage. Thus initially, the class $\theta$ consists of the singleton subsets of $\Gamma$, corresponding to the subscripts of each one of the specified special column vectors in $A$. 
To start with, find an initial basis $B$ for (1) which contains all the special column vectors as basic column vectors. This can easily be done by starting with the partial basis $\left\{A_{._{1}}, \ldots, A_{._{r}}\right\}$ and then completing it by standard pivotal operations of linear algebra. Performing the same operations on the right hand side column vector will help to find out whether $b$ lies in the subspace spanned by $\left\{A_{\cdot_{1}}, \ldots, A_{\cdot_{r}}\right\}$.

(i) If $b$ lies in the subspace spanned by $\left\{A_{._{1}}, \ldots, A_{r}\right\}$, i.e., if

$$
b=\alpha_{1} A_{\cdot_{1}}+\ldots+\alpha_{r} A_{\cdot r}
$$

and if it so happens that $\left(\alpha_{1}, \ldots, \alpha_{r}\right) \geq 0$, then every basis for (1) containing all the special column vectors is a desired basis and we are done.

(ii) If (12) holds but $\left(\alpha_{1}, \ldots, \alpha_{r}\right) \nsupseteq 0$, then obviously no desired basis exists.

(iii) Suppose $b$ does not lie in the subspace spanned by $\left\{A_{\cdot_{1}}, \ldots, A_{._{r}}\right\}$. In this case we continue.

3.2. At the end of 3.1 we have obtained a starting basis $B$ such that $v(B)=\left\{j: A_{j}\right.$ is a basic column vector in the basis $\left.B\right\}$ has a nonempty intersection with each subset in the class $\theta$ at this stage. The basis $B$ will be referred to as the current basis for this stage.

3.3. If it happens that the current basis $B$ is feasible to (1), (2), i.e., if $B^{-1} b \geqq 0$, then $B$ is a desired basis and we terminate. Suppose the current basis $B$ is not feasible to (1), (2), then $B^{-1} b \geqq 0$. Let $\nu(B)=$ $\left\{1, \ldots, r ; d_{r+1}, \ldots, d_{m}\right\}$ where $\left\{d_{r+1}, \ldots, d_{m}\right\} \subset\{r+1, \ldots, n\}$. Let

$$
\begin{aligned}
& \bar{b}=B^{-1} b, \\
& \bar{A}=B^{-1} A .
\end{aligned}
$$

The canonical tableau with respect to the current basis $B$ is

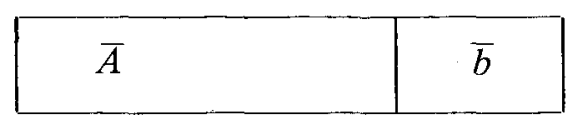


From this canonical tableau we see that the system of constraints (1) is equivalent to

$$
\begin{aligned}
& x_{i}+\sum_{j=r+1}^{n} \bar{a}_{i j} x_{j}=\bar{b}_{i} \text { for } i=1 \text { to } r \\
& x_{d_{i}}+\sum_{\substack{j=r+1 \\
j \neq d_{i}}}^{n} \bar{a}_{i j} x_{j}=\bar{b}_{i} \text { for } i=r+1 \text { to } m .
\end{aligned}
$$

By our assumption in (iii) of 3.1 , at least one of the $\bar{b}_{i}$ for $r+1 \leqq i \leqq m$ is nonzero. Let $L$ be the number of subsets which are in the class $\theta$ already.

Since the current basis $B$ is assumed to be infeasible to (1), (2), there must be at least one $i$ such that $\bar{b}_{i}<0$. For each $i$ between 1 and $m$, such that $\bar{b}_{i}<0$, let

$$
c_{L+i}=\left\{j: \bar{a}_{i j}<0\right\}
$$

For each $i$ such that $r+1 \leqq i \leqq m$ and $\bar{b}_{i}>0$ let

$$
c_{L+i}=\left\{j: \bar{a}_{i j}>0\right\}
$$

From (13) it is clear that every feasible basis $B^{*}$ for (1), (2) must satisfy $\nu\left(B^{*}\right) \cap c_{L+i} \neq \emptyset$ for each of the subsets defined in (14), (15). So we augment the class $\theta$ by adding all the subsets of the form $c_{L+i}$ obtained in (14) and (15), to it.

3.4. By our assumption, the current basis $B$ is not feasible to (1) and (2). So at the end of 3.3 we would have augmented $\theta$ by at least one subset of the form $c_{L+i}$ defined in (14). From (13) we notice that $\nu(B)$ has an empty intersection with any subset of the form $c_{L+i}$ defined in (14). Once we include a subset of the form $c_{L+i}$ in the class $\theta$ it remains there till the end. Also every basis $\widetilde{B}$ for (1) that we will consider later on will be required to satisfy $\nu(\widetilde{B}) \cap c_{p} \neq \emptyset$ for all $c_{p} \in \theta$ at that stage. Hence the current basis can never appear later on in the course of the algorithm. 
3.5. Now find a minimum cardinality subset $E$ of $\Gamma=\{1, \ldots, n\}$ which has a nonempty intersection with each subset of $\Gamma$ in the class $\theta$ at this stage. This is equivalent the familiar set covering problem for which various algorithm are discussed in $[1,2,3,11,13,14]$.

3.6. (i) If the cardinality of such a subset $E$ is $\geqq m+1$ then obviously no desired basis exists and we terminate.

(ii) Otherwise let the cardinality of such a subset $E$ be $\leqq m . E \cap c_{p} \neq$ $\emptyset$ for all $c_{p} \in \theta$ at this stage.

If the set $A_{E}$ is linearly dependent (this is easily determined by pivoting out), then let

$$
c_{E}=\left\{j: A_{._{j}} \text { is not in the linear subspace spanned by } A_{E}\right\} .
$$

The subset $c_{E}$ is also easily obtained when we check the linear dependence of $A_{E}$ by pivotal operations. Obviously if $\hat{B}$ is any basis for (1), then we must have $\nu(\hat{B}) \cap c_{E} \neq \emptyset$. So we augment the class $\theta$ by adding the subset $c_{E}$ to it and then go back to 3.5 to find a minimum cardinality subset of $\Gamma$ which has a nonempty intersection with every subset in the class $\theta$ at this stage.

On the other hand, if $A_{E}$ is linearly independent, then we obtain a basis for (1) containing $A_{E}$. (If cardinality of $E$ is $m$, then $A_{E}$ is itself a basis. Otherwise, $A_{E}$ is a partial basis and we obtain a basis by completing it, by pivotal operations.) We treat this basis as the new current basis and we repeat the same process with this new basis by going back to 3.3 .

3.7. A subset $E$ of $\Gamma$ obtained in 3.5 cannot appear in any stage later on when we execute the step 3.5 again because of the arguments in 3.6 and 3.4 .

Since there are only a finite number of subsets of $\Gamma$ and a finite number of bases of (1) all of which contain the special column vectors $A_{\cdot}, \ldots, A_{. r}$ as basic column vectors, the algorithm must terminate in a finite number of steps with either a desired basis or the conclusion that no desired basis exists when (i) of 3.6 is satisfied at some stage.

The efficiency of this algorithm depends on the efficiency with which the set covering problems encountered in the course of the algorithm 
can be solved and other things such as the number of set covering problems encountered. Limited computational experience with small problems seems to indicate that the number of set covering problems encountered before termination tends to be very small.

\section{Acknowledgements}

I wish to thank the referee for suggesting several improvements in the presentation.

\section{References}

[1] G. Andrew, T. Hoffman and C. Krabek, "On the generalized set covering problem," CDC Data Centers Division, Minneapolis, 1968.

[2] E. Balas and M.W. Padberg, "On the set covering problem," Management Science Research Report, No. 197, Carnegie Mellon University, 1970.

[3] M.L. Balinski, "Integer programming: methods, uses, computation," Management Science 12 (1965) $253-313$.

[4] A.V. Cabot and A.P. Hurter Jr., "An approach to zero-one integer programming," Operations Research 16 (1968) 1206-1211.

[5] G.B. Dantzig, Linear programming and extensions (Princeton University Press, 1963).

[6] F. Glover, "A note on linear programming and integer feasibility," Operations Research 16 (1968) $1212-1216$.

[7] R.E. Gomory, "The travelling salesman problem," in: Proceedings of IBM scientific computing symposium on combinatorial problem, White Plains, New York, 1964, pp. 93-121.

[8] I. Heller, "On the travelling salesman's problem," in: Proceedings of the second symposium in linear programming (National Bureau of Standards, Washington, D.C., 1955) pp. $643-665$.

[9] K.G. Murty, "On the tours of a travelling salesman," SIAM Journal on Control 7 (1969) $122-131$.

[10] K.G. Murty, “Adjacency on convex polyhedra," SIAM Review 13 (1971) 377-386.

[11] K.G. Murty, "On the set representation and set covering problems," University of Michigan, 1972.

[12] M. Raghavachari, "On connections between zero-one integer programming and concave programming under linear constraints," Operations Research 17 (1969) 608-684.

[13] R. Roth, "Computer solutions to minimum cover problems," Operations Research 17 (1969) 455-465.

[14] H.M. Salkin and R.D. Koncal, "A pseudo-dual all integer algorithm for the set covering problem," Technical Report Memo, No. 204, Operations Research Department, Case Western Reserve University, 1970. 